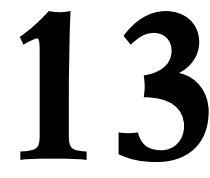

\title{
JOHN HENRY NEWMAN Y SU IDEA DE LA UNIVERSIDAD EN EL SIGLO XXI*
}

\author{
(JOHN HENRY NEWMAN AND HIS IDEA OF THE UNIVERSITY \\ IN THE 21ST CENTURY)
}

Miguel Rumayor

Universidad Panamericana. Escuela de Pedagogía

DOI: $10.5944 / e d u c X X 1.20088$

Cómo referenciar este artículo/How to reference this article:

Rumayor, M. (2019). John Henry Newman y su idea de la universidad en el siglo xxI. Educación XX1, 22(1), 315-333, doi: 10.5944/educXX1.20088

Rumayor, M. (2019). John Henry Newman y su idea de la universidad en el siglo XXI. Educación XX1. [John Henry Newman and his idea of the university in the 21st century]. Educación XX1, 22(1), 315-333, doi: 10.5944/educXX1.20088

\section{RESUMEN}

En este artículo se aborda la actualidad de algunos aspectos de la universidad en la filosofía educativa de John Henry Newman. Concretamente, el documento tratará de demostrar cómo las ideas del pensador inglés son válidas para reorientar la crisis conceptual en la que se encuentra esta institución en nuestros días. En primer lugar se criticarán, bajo el prisma de Newman, los problemas asociados al pragmatismo educativo que invade las aulas universitarias y la sola orientación de los alumnos hacia el mercado de trabajo. Por otra parte, se expone la importancia y la necesidad de la verdad en las aulas y el prestigio del profesor como guía comprometido en la búsqueda de la misma. Ambas ideas defendidas por Newman han desaparecido de muchas universidades occidentales de nuestro tiempo, con las negativas consecuencias que eso conlleva. Además, Newman propone la promoción y el desarrollo de la expansión de la mente de los universitarios gracias a las enseñanzas recibidas. Esta se dará en todas las disciplinas, 
con lo que este autor denomina como desarrollo del «hábito filosófico», el cual, como se explicará en este documento, consiste en orientar las materias más allá de sus dimensiones técnicas y de utilidad, creando en la mente de los alumnos una verdadera interdisciplinariedad de las ciencias. También se detallará en el escrito cómo, una vez que se empieza a alcanzar esta meta, objetivo de la universidad liberal que promueve Newman, los miembros de la comunidad académica podrán vincularse personalmente a la llamada gratuidad del saber y a los beneficios personales que otorga la vida académica. Estarán cimentando una verdadera alma mater universitaria, que se expandirá y ganará prestigio por el servicio que presta a la sociedad.

\section{PALABRAS CLAVE}

Universidad; filosofía de la educación; método educativo; profesión docente; trabajo intelectual; Newman.

\section{ABSTRACT}

This article addresses some current aspects about the university in the educational philosophy of John Henry Newman. Specifically, the paper will try to demonstrate how the ideas of this English thinker are valid in order to reorient the conceptual crisis in which this institution is nowadays embedded. From Newman's point of view, there is an initial discussion of the problems associated to the educational pragmatism that invades university classrooms, and the orientation of students towards the labor market. On the other hand, the importance and necessity of truth in the classrooms and the prestige of the teacher as a committed guide in search of it will be clarified. Both ideas defended by Newman have disappeared from many western universities of our time, with the negative consequences that this loss entails. In addition, Newman proposes the promotion and development of the minds of university students through the lessons they are taught. This will take place in every discipline through what this author calls the development of the "philosophical habit», which will also be explained in this document: an approach to subjects beyond their technical and useful dimensions, creating in the mind of the students a true interdisciplinary of sciences. Once this goal is achieved, according to the liberal university promoted by Newman, the members of the academic community will be able to personally link to the so-called free knowledge and the personal benefits of academic life. They will be building a true alma mater university, which will expand and gain prestige from the service it provides to society.

\section{KEYWORDS}

University; Philosophy of Education; Educational Method; Teaching Profession; Intellectual Work; Newman. 


\section{NEWMAN Y LA UNIVERSIDAD ACTUAL}

Algunos se preguntan si la universidad, una institución centenaria, tiene todavía algo que aportar a nuestra sociedad. Si lo que aprenden nuestros alumnos en las aulas está sirviendo para mejorar sus vidas en el sentido más profundo, haciéndoles mejores y personas felices, o por el contrario esta institución no añade — ni ha de hacerlo- nada al respecto y debe limitar su papel como capacitadora de trabajadores para el mercado laboral. Frente a este planteamiento, a lo largo de este escrito se analizarán algunos conceptos sobre la universidad de John Henry Newman (18011900) y su validez para nuestros días. Tales ideas fueron expuestas en diferentes documentos, algunos de especial relevancia como The Idea of a University (1907), The Rise and Progress of Universities (1909), y también en sus profundas reflexiones sobre la formación de la mente humana en $A n$ Essay in Aid of a Grammar of Assent (1903).

Newman se fundamenta en la Grecia clásica, donde se adivina el embrión de lo que en la Edad Media será el espíritu universitario. Pedagogos como Aristóteles o Platón fueron los fundadores de la Academia o el Liceo y se orientaron al desarrollo de un lugar de reflexión y encuentro entre profesores y alumnos. En tales instituciones imperan aspectos como la enseñanza de la causalidad, el valor del ocio y el uso superior de la inteligencia. Los que allí asistían se contagiaban de la necesidad de continuar enseñando y divulgando los conocimientos aprendidos (Himanka, 2015). En segundo lugar, Newman bebe del sistema formativo romano, más práctico y alejado de la reflexión teórica, dirigido a la formación del alumnado para su inmersión exitosa en la vida de la urbe. También lo hará de la llamada «universidad ecuménica» de la Edad Media (Fleischacker, 2009), como un espacio de análisis y debate entre el cristianismo y las nuevas corrientes filosóficas y teológicas llegadas a Europa por el norte de África. Por último, en Newman también se encuentra la idea de "universidad nacional», orientada al servicio de una comunidad determinada.

En el proyecto de la fundación de la Universidad Católica de Dublín, Newman trató de poner en práctica todas sus ideas, aunque no le fue posible, por eso después de algunos años de trabajo dimitió como Rector. Entiende la universidad como un espacio privilegiado e insustituible de la sociedad para la búsqueda de la verdad en todas sus dimensiones. El lugar donde los universitarios tienen que pertrecharse de las herramientas intelectuales, como primera finalidad, y de carácter, en segundo lugar, para trasformar en todas sus dimensiones la sociedad. Por eso para él allí se aprende el rigor analítico, el orden lógico, la generalización adecuada, la libertad de criterio, la disciplina exigente. Este proceso llevará a la mente a concentrarse en su objeto de estudio sin distracciones, sin prejuicios, sin fantasías ajenas al 
proceso intelectual, ya que «si un asentimiento es hecho sin suficiente base racional es un juicio temerario, un capricho o un prejuicio» (Newman, 1903, p. 258).

Para Newman la vida académica no debe caminar al margen de la sociedad, convirtiendo los foros universitarios en concilios de eruditos especializados que solo saben hablar de su ciencia y no se mezclan con los intereses y las necesidades de sus conciudadanos. Si la universidad no está en consonancia con la sociedad no sirve para nada, por eso ha de preparar gente para lo que esta necesita. Ahora bien, la pregunta que se hace Newman, como más tarde lo hizo Ortega y Gasset en su libro Misión de la Universidad (2007), que también podemos trasladar a nuestros días (Torralba, 2013), es sobre lo que la sociedad realmente necesita.

Newman percibe que la moderna división del trabajo, defendida entre otros por Adam Smith, ha originado también la fragmentación del ser humano (Culler, 1958). Así que cuando lo único que se puede ofrecer es la obtención de un título que posibilite la penetración con éxito en el mercado laboral, la vida académica pierde su virtualidad como positiva trasformadora de la sociedad. Como ocurre en nuestros días, la universidad camina detrás del mercado a la hora de articular sus titulaciones y de promover proyectos de investigación, haciendo, directa o indirectamente, que sus alumnos se conviertan en individuos carentes de sentido crítico y se plieguen sin resistencia a la gran maquinaria de la producción y el consumo. Cuando el criterio de empleabilidad y especialización pone a la universidad al servicio del mercado de trabajo, la vida académica se trasforma en una formación profesional de grado superior y se ignora la potencialidad creativa de la aulas universitarias. Para Newman (1907) en la medida en que el conocimiento se estrecha y hace particular deja de ser sabiduría para la persona y para la sociedad.

Todo lo dicho nos lleva a replantearnos la validez del modelo actual de universidad, al menos aquel que propone el Plan Bolonia, que trata de ser una copia del modelo americano sin iguales recursos físicos y humanos (Nubiola, 2008). A la sombra de tal modelo, repleto de prácticas y actividades en clase, los alumnos no son formados para expandir sus mentes ni pensar por sí mismos, sino solamente entrenados para desarrollar técnicas medibles, por tanto, en el sentido de Newman, poco formativas (Luque, 2016). Para el intelectual inglés todo conocimiento que no produce acciones inmanentes, cambios profundos en la persona, no forma realmente. Solamente por el crecimiento de estas, la persona va más allá de los objetos conocidos y el ser humano podrá tener después una auténtica proyección vital, una vida creciente capaz de abandonar el límite mental y proyectarse irrestrictamente 
ante cualquier realidad, tal y como fundamenta Leonardo Polo (1998) desde una reflexión epistemológica.

Además hay que preguntarse qué papel juega el profesor en este sistema. Como más adelante se explicará, colocar en la cima de la formación personal la posibilidad futura del enriquecimiento económico del alumno conlleva devaluar el magisterio universitario, ya que implícitamente supone mostrar la figura del profesor de tiempo completo como un «refugiado intelectual». Alguien incapacitado para enfrentarse a la producción y al mundo real, que encontró en las aulas un espacio para enseñar lo que otros hacen mucho mejor en la práctica, como es ganar dinero. Unido a eso la institución universitaria no es ajena al tsunami que ha supuesto la llegada a su mundo de las nuevas tecnologías y particularmente la universalización del acceso a las fuentes del saber. Hoy en día los alumnos pueden contrastar en tiempo real si la información que se está dando en clase es veraz y conocer la fuente en las que el académico se ha fundamentado. Pueden, por ejemplo, iniciar después de la clase —en ocasiones lo hacen durante la mismauna conversación en una red social con una persona o grupo de personas, tal vez con alguien que sabe más que el profesor sobre el tema. También complementar, criticar o trasferir lo aprendido en clase a otros mejores expertos. Para muchos el acceso universal a la información del alumno y el campo de discusión se ha convertido en inabarcable, provocando que el papel del profesor universitario esté en tela de juicio, algunos se preguntan si su función se ha reducido a un "intermediador intercultural» (Reyero, 2014, p. 128). Frente a esto Newman también ofrece valiosas reflexiones.

\section{LA VERDAD Y EL PRESTIGIO EN EL PROFESOR UNIVERSITARIO}

Uno de las ideas fundamentales de Newman sobre la educación reside en que el universo es objetivo e inteligiblemente unificado (Rupert, 1998). Por eso, gracias al sentido "sentido ilativo» (Newman, 1903) existente en todas personas la verdad puede ser conocida. Todos deseamos la verdad de lo concreto (Newman, 1903), ya que tal conocimiento, una vez que se lleva a cabo, contribuye en muchas ocasiones, en cuanto que puede incidir directa o indirectamente sobre la voluntad, a hacer bueno al que lo recibe. Esta acción cognoscitiva se produce por lo que Newman llama acto de asentimiento real, el cual, al margen de su dimensión de utilidad tangible, libera al hombre y le llena de sosiego y paz interior (Newman, 1903). El saber auténtico perfecciona la propia naturaleza. La mente humana anhela profundamente la verdad como su objeto propio, alcanzarla satisface inequívocamente su propia constitución natural (Newman, 1907). Así se comprende que el teólogo inglés afirme que no puede existir la universidad sin la búsqueda de la verdad. Newman cree que verdad y formación personal son indisociables. 
Sin embargo, la verdad en nuestra academia ha quedado subjetivizada, aherrojada al ámbito de la percepción individual. La reducción de todo aprendizaje a su dimensión práctica es tal vez una consecuencia que procede en gran medida de un intento de paliar la carencia asociada a ese planteamiento. La supuesta neutralidad de la verdad, tan extendida como un mantra por la sociedad, conlleva una falsificación de la misma vida universitaria, una etiqueta extraña a su esencia. Para Newman, en el ámbito académico, como en otros de la vida, el ser humano al poseer conciencia no puede enfrentarse a la búsqueda de la verdad desde un plano personal distante y distanciado de la misma, tal y como defiende el relativismo. El contacto intelectual con esta no deja indiferente a la persona. La esencia de la vida universitaria se fundamenta en la capacidad del hombre de asentir realmente a las verdades encontradas.

Quizá sean comprensibles ciertas reacciones relativistas en la vida universitaria. No se trata de que el profesor se convierta en una especie de sacerdote de la verdad; su valor reside más bien en actuar como testimonio viviente de esa empeñada búsqueda, ya que «si queremos formarnos plenamente en un rama del saber con su diversidad y complicación debemos consultar a un hombre vivo y escuchar su voz viviente» (Newman, 1909b, p. 8). La verdad va más allá de la acumulación de datos o de informaciones, de ahí que los alumnos necesiten tener un guía claro que les ayude. Por eso para Newman la trascendencia del profesor universitario consiste en que es alguien capaz de impulsar al alumno a una decidida búsqueda personal de la verdad. Un buen profesor no es tal por lo que enseña sino más bien por lo que invita a aprender (Reyero, 2014).

Tristemente, hoy en día, el docente universitario en muchos espacios académicos no puede mostrar públicamente, ni con sus compañeros ni sus alumnos, una abierta preocupación por este tema. En algunas universidades hacerlo le desacredita automáticamente y le convierte en un fanático, ya que las enseñanzas tienen que ser siempre asépticas y desvinculadas de cualquier planteamiento ético relacionado con la búsqueda de la verdad. Cuando por absoluta necesidad el docente tenga que hacer un juicio, habrá que acudir a los tópicos al uso de lo políticamente correcto. Esto va a provocar, sobre todo en el campo de las humanidades y las ciencias sociales, directa o indirectamente, que el trabajo del académico no se dirija al estudio y a la exposición rigurosa de los temas, con la idea de ofrecer desde su ciencia una solución a las necesidades verdaderas que la realidad plantea a cada momento. Por el contrario, estará preocupado en constatar que sus investigaciones estén alineadas al main stream, la corriente de pensamiento en boga, que a veces consiste en una combinación, supuestamente académica, de los tópicos al uso y de corrección política. 
Además, en un modelo de universidad como el que ofrece la Declaración de Bolonia (1999) en donde no se promueve la búsqueda de la verdad, tal y como se refleja en algunas instituciones de nuestros días, la figura del profesor no tiene sentido. Su función se limita a la trasmisión de una serie de informaciones sin unidad interna, especialmente cuando hay una renuncia explícita al fundamento universal de las ciencias, haciendo en última instancia que estas se puedan relacionar entre sí. Para Newman (1903) un saber fragmentado, como el que se ofrece en muchas universidades, es un saber sobre el que la persona no puede realizar un asentimiento real, principio de toda formación humana. Si las nociones aprendidas no residen en verdades internamente articuladas, es normal por tanto que su sentido no comprometa casi a quien lo recibe. Frente a esto Newman ofrece uno de los principios metafísicos sobre el que gira su liberalismo educativo: formar a la persona desvinculándola de todo menos de la verdad que encuentra en su conciencia cuando esta se contrasta con lo real. El ser humano, gracias al ejercicio de la racionalidad, y también con la ayuda de la luz de su conciencia, será capaz de conectar personalmente el sentido de las ciencias aprendidas entre sí (Mulcahy, 2008).

$\mathrm{Ni}$ en la vida personal, ni en la vida corriente, la verdad puede ser completamente poseída por nadie, por lo que todos los que forman parte de la comunidad universitaria, en diferente medida, tendrán una visión limitada y parcial de la misma. De ahí que la disposición del maestro, alejada de todo dogmatismo e imposición, debe ser la de un humilde y empeñado amador de la misma (Ahedo Ruíz, 2016). Tal actitud, como refleja la vida del mismo Newman, mostrada ante los alumnos en las diferentes tareas universitarias, tiene una enorme influencia en ellos (Waldmann, 1967). Gracias a esta se puede contagiar la veneración personal en la que se apoya el prestigio de cada ciencia. Entender la academia de esta manera supone construir una verdadera comunidad de aprendizaje en la que todos, cada uno a su nivel, se esfuerzan por ser cooperadores de la verdad. Es triste apreciar cómo el tedio existencial de los ciudadanos de la sociedad occidental, imbuidos de relativismo, ha corrido de la mano con la crisis contemporánea de la universidad. Hay que recordar aquí la idea de Newman (1903) cuando afirmaba que por los dogmas hay gente que muere, pero nadie lo hará por una conclusión. En el amor a la verdad radica el prestigio personal y profesional del profesor sobre sus alumnos, de ahí que sea una herramienta insustituible para la formación de universitarios. La búsqueda sincera, apasionada y trabajosa de la misma provoca la adhesión y confianza de los que están siendo formados. Frente a lo que defiende la difusión del racionalismo en las aulas, para Newman las personas no solamente crecemos intelectualmente cuando entendemos las cosas sino, sobre todo, cuando confiamos en quien tenemos delante. Los alumnos se 
ponen interiormente en manos de profesores que tienen, o buscan tener, una relación personal y auténtica con la verdad de lo que enseñan.

\section{LA EXPANSIÓN DE LA MENTE Y EL HÁBITO FILOSÓFICO}

Uno de los mayores hándicaps con los que nos encontramos hoy en día los profesores universitarios es que los alumnos se concentren en la clase y en realizar lo que se les pide. La llamada generación Millenial es un grupo social distraído y le cuesta un gran esfuerzo centrar su reflexión en un solo objeto de trabajo. Esto hace que, desafortunadamente, nuestros alumnos universitarios muchas veces al final de sus estudios no hayan aprendido una cosa bien sino muchas mal. Pasan horas, en el colegio primero y en la universidad después, leyendo con el profesor lo que aparece en el Power Point, y escuchando peroratas interminables mientras sus cabezas se hallan en la nube, tal vez cavilando la respuesta más ingeniosa para un chat. Esto es un problema, ya que la capacidad de concentración en soledad y la reflexión serena son algunas de las necesidades fundamentales para el desarrollo del liderazgo social, como ha puesto de manifiesto Willian Deresiewicz en su famosa conferencia Solitude and Leadership, pronunciada en el año 2009 frente a los cadetes de West Point.

Nuestro autor entiende que el conocimiento de las cosas puede ser dirigido voluntariamente y alcanzar cada vez mayor precisión, accuracy of thought lo denomina (Newman, 1907, p. 501), una meta esencial para la universidad de nuestros días. La formación de la mente en la vida universitaria se concreta en los hábitos de firmeza, sutileza, capacidad de entendimiento. No conlleva solamente un cambio profundo en la inteligencia del alumno, sino que supone también de modo indirecto la formación de su carácter (Franco, 2004). Compara Newman (1907), metafóricamente, el crecimiento de la mente con el proceso de la digestión de alimentos. La corriente pedagógica del Critical Thinking ha desarrollado recientemente mucho más lo que Newman ya pensó en el siglo XIX. Para él, si no conseguimos hacer subjetivamente nuestros los objetos intelectuales aprendidos, de modo que comiencen a formar parte sustancial de nuestra vida intelectual, no podemos hablar de ninguna forma de la ampliación intelectual en la persona. Además es allí, y no en otro sitio, donde se construye verdaderamente la vida universitaria: en la cabeza de los profesores y los alumnos. Los primeros como formadores en una ciencia y los segundos como receptores, establecen un diálogo entre sus mentes y el mundo, la tradición, la técnica, el progreso, etc. (Newman, 1909b). Como ha señalado Dunne (2015), parece claro que un sumado de datos, de puntos de vista y de realidades sin más, sirve de poco para comprender con hondura las cosas y hacer una persona verdaderamente pensante. 
Así que la capacidad de razonar no está ni formada ni en proceso de hacerlo sin que de forma activa se busque la verdad. Tal expansión de la mente, también llamada por Newman «conocimiento filosófico, crecimiento de la mente o iluminación» (1907, p. 125), equivale a la construcción de un hábito filosófico. Sin embargo Newman no pretendió crear una universidad de filósofos contemplativos, imprácticos y alejados del mundo. Al hablar de la formación filosófica de los alumnos universitarios incluye a todas las ciencias sin discriminar ninguna. Tampoco excluye la preparación para el desarrollo de una profesión concreta (Esteban Bara, 2015), por eso entiende que el aprendizaje de ciencias como la física: «exalta, agranda y tranquiliza la mente (...), al sacarla de sí misma destruye la ansiedad y le proporciona objetos de admiración» (Newman, 1907b, p. 256).

Que todos los conocimientos tengan un trasfondo filosófico no significa que en clases de química orgánica o de estructuras metálicas, por ejemplo, el profesor deba descolgarse con una sesuda reflexión sobre Kant o Hegel. Newman no trata de trasformar cualquier tipo de enseñanza universitaria en una extraña filosofía (Ker, 2011). Quiere decir, por el contrario, que el académico debe de tener en su mente siempre una explicación de fondo con sentido para relacionar, acentuar, delimitar, ejemplificar y hacer ver la articulación interna entre los conocimientos que se están exponiendo en la clase. Esto es educar la mente en la universidad para formar un gentleman (Newman, 1909b, p. 10). Crear un hábito filosófico en los alumnos, hombres y mujeres, supone desarrollar en ellos la capacidad de admiración frente a las realidades que les rodean y especialmente hacerlo en la ciencia en la que están desarrollando sus aprendizajes (Ahedo Ruíz, 2016). Supone desarrollar con excelencia, mediante la argumentación, el estudio, la discusión, la defensa de ideas, etc., las virtudes intelectuales. Este modelo universal es compatible con el que personalmente Newman aspira, el del «gentleman cristiano» (Tillman, 2008, pp. 13-16), poseedor de tales hábitos intelectuales además de estar impregnados de la humildad, la caridad y la fe cristianas.

Para Newman, tal y como sucedía en la paideia griega, se trata de la formación de un hábito para toda la vida (Rupert, 1998). Como explica A. McIntyre (2009), consiste en haber crecido en la capacidad de elaborar un razonamiento crítico eficaz, el cual siempre estará orientado de manera práctica hacia fines concretos, tiene en cuenta la mayoría de las circunstancias que confluyen en la realidad y dispone sabiamente de los medios en relación a los fines. En cierto modo se trata de pensar de forma aristotélica, ya que, según Newman (1903), pensar así es la forma más adecuada de hacerlo. Aquí radica también el sentido auténtico de la interdisciplinariedad universitaria, el cual se llega a crear, con mucho 
trabajo y dedicación, en las mentes del profesor y posteriormente en sus alumnos.

En su origen, en la universidad medieval, en el avance de los diferentes grados que en ella se obtenían, se encontraba implícito el desarrollo del conocimiento de una determinada ciencia y también el crecimiento en la capacidad de comunicarla con éxito a los alumnos, las palabras «maestro» $\mathrm{y}$ «doctor» eran intercambiables por la de profesor (Schulman, 1986). Por eso en el modelo de universidad que defiende Newman un profesor debe ser un auténtico maestro, gran conocedor de un área de conocimiento (Fleischacker, 2009). Para él no puede existir un buen docente sin un compromiso claro con el estudio constante y la investigación rigurosa (Ker, 2011). No puede ser de otro modo, ya que un académico investigando contrasta y refresca sus conocimientos, lo cual le facilitará, junto con el desarrollo de habilidades propias de la docencia, oratoria, didáctica, etc., la posibilidad de exponer brillantemente los temas a sus alumnos.

Finalmente, la expansión de la mente propia de la vida universitaria se sitúa para Newman entre varios parámetros. Por una parte se trata de sobrepasar todo lo que podría denominarse como «cultura de la opinión». Es decir, ayudar a la mente, mostrándole el placer personal intrínseco que la búsqueda de la verdad de suyo comporta (Newman, 1903), para que intencionalmente acuda a las fuentes del conocimiento sin que asuma superficial e inmaduramente lo que reciba de otros (Luque, 2016). Ya que: «si nadie envidia a un loco por el brillo y variedad de sus conceptos, ¿por qué debíamos exaltar el cultivo de un intelecto presa no solo de fantasías estériles sino también de hechos estériles, de intrusiones arbitrarias desde afuera?»(Newman, 1907, p. 141). De modo que expandir la mente es contrario al memorismo o la acumulación excesiva e inconexa de datos o de información. Rechaza la memorización sin sentido o la erudición vacía, ya que, al igual que aprecia Ramón y Cajal en Los Tónicos de la voluntad. Reglas y consejos sobre la investigación científica (2015), frente al famoso dicho: el saber sí ocupa lugar.

\section{UN MODELO DE EDUCACIÓN LIBERAL PARA LA UNIVERSIDAD}

La educación liberal se puede concebir de dos maneras, según se haga desde un plano ideológico y político o desde una posición filosófica y metafísica, tal y como lo hace Newman. En la primera óptica, liberal es aquel tipo de educación plural, facilitado por un modo de sociedad y un sistema (Minogue, 2005), que ofrece al individuo la capacidad de determinar y decidir el componente de una realidad dada. Se trata de un liberalismo educativo tanto en los medios como en la determinación de los fines. El 
segundo modelo de educación liberal, el de Newman, asume, igual que el primero, una variedad de metodologías válidas en el proceso educativo, ahora bien, la meta filosófica de todas ellas sería que la persona aprehenda el bien moral y busque la verdad por el valor que tiene en sí misma. Al buscar estas realidades la mente se libera de todo, de ahí el adjetivo liberal, por ello tal educación sería contraria a la instrucción servil (Mlinar, 2013). Así, Newman no busca formar al ser humano en un entramado de ideas para tener éxito y ganar dinero, sino para que adquiera un más profundo entendimiento del mundo que le rodea. La búsqueda de la sabiduría, centro de la educación liberal de este pensador, consiste en el deseo de encontrar lo permanente en las cosas, aquello que persiste en la realidad que nos rodea y que se encuentra más allá de los accidentes y de la utilidad práctica de lo real.

El liberalismo educativo de Newman sostiene que el conocimiento debe de ser buscado por el valor que tiene en sí mismo. Es decir, para Newman hay cosas que merece la pena saber y otras no. Un intelecto educado se dirige a las primeras e ignora o desprecia las segundas. Tal intelecto tiende a la sabiduría. La consecuencia es que es responsable en su búsqueda de información. No navegará a la deriva en cualquier realidad, o por cualquier página web diríamos ahora, ni se dejará nunca absorber por un conocimiento determinado (Esteban Bara, 2015, p. 9) que le bloquee o le aleje de su búsqueda. Para Newman toda verdadera educación o es liberal o no es educación en absoluto. Es decir, o las cosas que se aprenden están destinadas a producir una transformación interior y definitiva en la mente, y no dependen de la utilidad práctica de lo aprendido, o por el contrario, no estaríamos hablando de educación propiamente sino de instrucción o de entrenamiento (Newman, 1907, p. 114). La consecuencia sería que una institución sin un ethos moral no puede ser denominada como tal universidad, sino que se trataría más bien de un colegio de adultos para el beneficio económico del estado (Chapman, 2011, p. 227).

De ahí que cuando Newman utilice la palabra cultura lo haga en un sentido diferente al actual (Ker, 2011, p. 22). No se trata de una acumulación de datos o experiencias, sino que para él la cultura es sobre todo «cultivo de la mente». El fin de la universidad liberal no es el desarrollo de una serie de capacidades. Por muy elevado que sea este en su crecimiento en las aulas se trata de un fin limitado. Tampoco lo es la adquisición de ciertas competencias para una entrada exitosa en el mercado de trabajo, tal y como propone la mencionada Declaración de Bolonia. La tarea fundamental de la universidad es el desarrollo de una actividad sobre el alumno, la cual, si se produce equivale a una trasformación personal definitiva.

Algunos autores consideran que el gran error de la educación liberal de Newman consiste en proponer un modelo articulado en un telos, una 
finalidad universal en el desarrollo de la naturaleza humana. La crítica de esta idea hacia Newman parte de una concepción autónoma e inmanente de la libertad personal, cuya finalidad es la que cada sujeto inventa y decide de cara a su propia realización personal (McIntyre, 2013). Frente a esta postura otros autores contemporáneos consideran, en la línea de Aristóteles y Newman, que la educación, particularmente la universitaria, tiene como meta dar plenitud a la naturaleza humana (Ibáñez, 2017). Hay que matizar que la idea liberal que propone Newman, y que otros autores como el poeta T.S Eliot divulgaron en su tiempo, o más recientemente ha difundido Josef Pieper, no versa en la generalización de una práctica personal de unas creencias religiosas determinadas, como erróneamente ha afirmado Kenneth McIntyre (2013) acerca del pensamiento de Newman. El ideal universitario de Newman no consiste en extender al gran público su fe cristiana, ni tampoco, a pesar de su condición de sacerdote católico, considera a la universidad directa y fundamentalmente como un instrumento misionero de la Iglesia.

Por último, el liberalismo educativo de Newman no se dirige a que el individuo se desvincule de la relación con los demás. Tal interpretación del liberalismo, distinta a la de nuestro autor, tiene su origen en la estructura social creada a partir de la concepción religiosa del libre examen de Lutero, que a su vez dará paso al cogito cartesiano y a toda la Modernidad. Lutero propugna la soberanía y la distancia absoluta del "yo», de la conciencia individual, frente a la vida y la realidad de los otros. Es normal que desde ese planteamiento liberal, totalmente diferente al de Newman, se conciba la universidad como un espacio social valorativamente neutral y aséptico (Esteban Bara, 2013). Por el contrario, la educación liberal de Newman es compatible con la idea de universidad como comunidad en su sentido más pleno. Sus miembros no forman parte de ella solamente bajo un modo de asociación temporal o de intereses individuales, como ocurre en muchas asociaciones modernas, sino que se implican personalmente en sus tareas con sus intelectos, voluntades, sentimientos, etc. Se trata de una comunidad de aprendizaje en donde las grandes ideas de la historia del pensamiento se comparten y analizan (Newman, 1907). Donde profesores y alumnos viven la ilusión de mirar con profundidad la disciplina que aprenden (Torralba, 2013, p. 70). Por eso no es extraño que en esta comunidad se produzcan amistades sólidas entre sus miembros, entre los profesores y también con los alumnos.

\section{LAS HUMANIDADES Y EL PAPEL DE LA TEOLOGÍA NATURAL EN LA UNIVERSIDAD DE HOY}

Tristemente forma parte del discurso social de nuestros días decir que las humanidades no aportan nada útil a la sociedad y que por tanto 
estudiarlas equivale, de una forma u otra, a perder el tiempo. No deja de ser curioso que para sostener esa idea haga falta asumir algunos presupuestos que de ninguna manera justifican las ciencias experimentales, como son, entre otros, el sentido que por sí mismo tienen las cosas o qué significado encierra la idea de aprovechar el tiempo. No es posible prescindir de las humanidades, porque afloran de forma natural entre las personas para cualquier proyecto de vida que trascienda de una visión mecánica de la realidad. Desde el otro extremo también hoy existen los que consideran a la humanidad, y por tanto a las humanidades, como un proyecto construido al propio gusto. Lo humano, lo «demasiado humano» diría Nietzsche, se fundamenta únicamente en la libertad prometeica de afirmación individual, lo voluntariamente decidido. Aquí también sucede que las humanidades dejan de tener valor por sí mismas y se convierten en proyecciones voluntaristas sobre aquello que, aparentemente, puede afirmar soberanamente la propia vida.

Como se decía antes, para Newman (1907) no se puede plantear una auténtica formación académica sin una visión universal de la realidad y de la naturaleza humana. Así, el supuesto moral de situar los valores universitarios en el consenso social, como está sucediendo en nuestros días, es un grave error. Consiste en dirigir el peso de la formación moral y la jerarquía de valores por el mercado o las modas sociales y no por la esencia de las cosas. La verdad es el objeto de las aulas universitarias, no solamente la que nos ofrecen las ciencias experimentales, sino las verdades sobre el hombre que muestran las humanidades, insustituibles para organizar la vida entre las personas.

Por ello Newman (1909b) entiende que el valor que encierran las humanidades para la sociedad es indeclinable. Una sociedad fragmentada en multitud de relatos minúsculos, de costumbres y de ideologías diversas, ofrece un rompecabezas social que hace imposible pensar en la concentración y ordenación de la importancia de saberes conforme a la verdad del hombre, el bien y la belleza de lo humano. Este multiculturalismo, enseñado en muchas aulas, entendido como la perspectiva de neutralidad moral ante todas las tradiciones, desarraigado internamente de la tradición a la que pertenece cada sociedad, no permite comparar profundamente las virtudes y defectos de la propia cultura con los de otras y viceversa. Desde tal presupuesto es imposible juzgar movimientos políticos como el nazismo, la tortura o la violencia de género. La reducción de la vida universitaria al ámbito de lo útil o de lo socialmente práctico, sin las humanidades, hace que los que participan en el proyecto académico no puedan plantearse de alguna manera formar a los alumnos en elementos como la dignidad personal, o, como hizo Newman en la Carta al Duque de Norfolk (1900), mostrar el verdadero sentido de la libertad de conciencia en relación a la 
autoridad de Roma y al poder del estado inglés. Tales aspectos y algunos otros son un bien en sí mismo y tanto su existencia como su captación solo pueden ser entendidos en la estructura de gratuidad que ofrecen las humanidades (Newman, 1907). Por eso tal vez habría que comenzar desde la educación infantil a desarrollar las ideas de Newman (Franco, 2004), ya que las humanidades son tan necesarias como las ciencias empíricas y no son fruto de la visión social y cultural de una determinada época, gracias a ellas podemos hacer juicios morales universales.

Otro elemento necesario para la vida universitaria es la teología natural, tal y como la entiende Newman, esto es, no como enseñanza de la religión o teología católica sino como conocimiento de Dios, como fundamento último del universo. Se trata del estudio y la concepción del saber y el desarrollo humano radicalmente fundamentado, aspecto que no es lo mismo que situarlo bajo supervisión eclesial (Newman, 1908b). Para nuestro autor el estudio de tal teología no es más que un paso superior en el desarrollo filosófico, aquella segunda instancia que llevó a Aristóteles, entre otras cosas, a adentrarse en una reflexión racional sobre el origen de la contingencia del ser, de los grados de perfección de lo natural y de la causación inscrita en la naturaleza.

Newman, frente a lo que algunos afirman (McIntyre, 2013), considera que para que haya teología natural en las aulas universitarias no es necesario un consenso social, idea defendida por autores como John Rawls y Richard Rorty. El planteamiento universitario de Newman para concebir una formación académica llevada a cabo hasta sus últimas exigencias racionales es el aristotélico: Dios como la causa última de la realidad. Por eso, la teología natural universitaria en Newman no es otra cosa que la búsqueda sincera de principios últimos unificadores del saber (Hütter, 2013). El Dios que debe ser estudiado en las aulas universitarias es aquel al que ha tenido siempre acceso el ser humano con su razón natural, que los pensadores medievales situaban en el ámbito de los llamados preambula fidei. También, desde el punto de vista de la formación religiosa que puede ser impartida en la universidad, Newman defiende el uso de la razón y las herramientas que esta otorga para fundamentar la propia fe (Newman, 1909), ya que para él la fe posee un componente intelectual, no racionalista, por el que se llega a conocer una realidad concreta (Mlinar, 2013).

Por eso, desde la óptica de Newman, ubicar la teología natural en el ámbito del sentimiento personal o de la creencia supersticiosa equivale a privar a las ciencias de su fundamentación última (McIntyre, 2012), ignorando que la universidad es un espacio de búsqueda de la universalidad. La dispersión y falta de unidad curricular universitaria a la que asistimos en nuestros días y la separación sin relación alguna entre las diferentes ramas 
del saber, fuera de su convergencia en aspectos de tipo técnico, proviene del prejuicio intelectual de creer que no existe una relación universal entre todas las cosas (McIntyre, 2009). Consecuentemente, la unidad, el sentido y la relación causal solo se buscarán en la propia ciencia. Solo cabrá concebir ciencias que actúan aisladamente, enseñadas por hombres de mente estrechamente especializada, los cuales no están abiertos a la unidad y el fundamento de lo real en el que en última instancia confluyen todos los saberes.

\section{LA NECESIDAD DE VOLVER AL ESPÍRITU UNIVERSITARIO Y LA UNIVERSIDAD COMO ALMA MATER}

A modo de conclusión se puede decir que toda formación verdadera es educación para la vida y no para un solo aspecto de la misma como es el trabajo. Cuando la orientación académica no gira en torno a esta idea, cautiva la vida intelectual, y hace que la universidad, como está sucediendo en todo el mundo, someta sus currículos y planes formativos a los vaivenes del mercado laboral y a la ley de la oferta y la demanda. En vez de influir en la sociedad lentamente con modelos más humanos, profundos y completos de vida, se coloca a la escucha, cuando no a las órdenes, de la gran maquinaria de producción de trabajadores, egresando personas muy especializadas, interiormente formateadas, con dificultad para desarrollar un proyecto personal exitoso. Newman, como se ha visto a lo largo de este escrito, rechaza la súper especialización de la academia, que no es incompatible con la dedicación al estudio de una disciplina. Por eso en su pensamiento «tanto el generalismo superficial como la estrecha especialización reflejan una mente no educada» (McIntyre, 2009, p. 348). Es decir, para el filósofo inglés es importante conocer una ciencia con claridad y no tener un intelecto disperso en cientos de detalles minúsculos, carentes de unidad. Pero no quiere convertir la universidad en una especie de barniz intelectual para la inserción en la vida profesional. Lo que busca Newman es que a partir del estudio serio de una disciplina la persona sea capaz de formarse en un hábito duradero de la mente que le ofrezca orden en su pensamiento, rigor, flexibilidad y apertura para enfrentarse a lo real.

Así, se puede concluir de lo dicho que muchas universidades necesitan replantearse su espíritu universitario, para que de este modo la universidad se convierta en un alma mater, una madre nutricia para las mentes y los corazones de los que allí estudian, tal y como la entendía Newman, ya que algunas instituciones ni siquiera saben si tienen o han tenido alguna vez tal espíritu. Este se forma en la comunidad de profesores y alumnos en las diferentes disciplinas del saber, que se congregan alrededor de la búsqueda de la verdad (Newman, 1909b). Es el espíritu universitario el espacio 
interior que nace en cada universitario al que, pasado el tiempo, la persona siempre vuelve con enorme nostalgia y agradecimiento por lo recibido (Miller, 2011). No se trata de un concepto solo asequible a historiadores o para estudiosos del medievo. Por el contrario, hablamos del meollo del que nace la vida universitaria, el único ámbito humano de donde brota la academia. Así sucedió en la vida de Newman (1908) y su paso por el Trinity College de Oxford y como Fellow en Oriel College, donde a pesar de los sufrimientos que tuvo que padecer en su itinerario hacia su conversión al catolicismo, los cuales le empujaron a abandonar este lugar, siempre guardó un profundo afecto y agradecimiento (Newman, 1908). Como decimos, cuando dicha comunidad existe, se deja entonces una huella indeleble en la mente de los que allí han participado, un asentimiento real sobre un bien espiritual de valor incalculable (Miller, 2011). Es normal por eso que aparezcan lazos de unión profundos entre la institución y cada uno de los miembros pertenecientes a ella (Newman, 1907). Los promotores de las universidades, privadas y públicas, no tendrían que invertir tanto dinero en marketing para promover sus universidades si entendieran el valor que para los universitarios tiene todo esto. Cuando una universidad posee un espíritu sólido, imbuido en el corazón y la mente de sus alumnos, entonces no hay dinero suficiente para pagar la propaganda vital que siempre hacen los que de él han participado.

\section{NOTA}

1 Este artículo es el resultado de una investigación totalmente financiada y desarrollada en el National Institute of Newman Studies, Duquesne University, Pittsburgh, EE.UU. 


\section{REFERENCIAS BIBLIOGRÁFICAS}

Ahedo Ruíz, I. (2016). La universidad: una escuela al servicio de la verdad. Revista Complutense de Educación, 27(2), 517-532. doi: dx.doi.org/10.5209/ rev_RCED.2016.v27.n2.46604

Chapman, M. (2011). Newman and the Anglican Idea of a University. Journal For the History of Modern Theology, 18 (2), 212-227. doi: d o i . org/10.1515/ZNTH.2011.010

Culler, D. (1958). The Imperial Intellect. Massachusetts: Yale University Press.

Deresiewicz, W. (2010). Solitude and Leadership. Recuperado de https://goo. gl/DRLoQ6

Dunne, G. (2015). Beyond critical thinking to critical being: criticality in higher education and life. International Journal of Educational Research, 7, 86-99. doi: http://dx.doi.org/10.1016/j. ijer.2015.03.003

Esteban Bara, F. (2013). El profesor universitario y su quehacer docente: la perspectiva comunitaria. Revista Española de Pedagogía, 71(255), 227242.

Esteban Bara, F. (2015). Wilhelm Von Humboldt, Cardinal John Henry Newman and José Ortega y Gasset. Some Thoughts on Character Education for Today's University. Journal of Character Education, 11(1), $1-20$.

Fleischacker, D. (2009). From Athens to Dublin: John Henry Newman on the History of the University. Newman Studies, 6(2), doi: 10.5840/nsj2009624

Franco, P. (2004). Liberal Education and the Good Society. The Good Society, 13(3), 54-58. doi: dx.doi.org/10.1353/ gso.2005.0019.
Himanka, J. (2015) On the Aristotelian origins of higher education. Higher Education 69(1), 117-128. doi: dx.doi. org/10.1007/s10734-014-9764-7

Hütter, R. (2013). University Education, the Unity of Knowledge -and (Natural) Theology: John Henry Newman's Provocative Vision. Nova Vetera, 11(4), 1017-1057.

Ibáñez-Martín, J.A. (2017). Horizontes para los educadores. Madrid: Dykinson.

Ker, I. (2011). Newman's Idea of a University and its relevance for the 21st Century. Australian eJournal of Theology, 18 (1), 19-31.

Lorda, J L. (2014). La educación, el arte de pensar. Revista Española de Pedagogía. 72(258), 315-325.

Luque, D. (2016). La influencia de John Henry Newman en la reflexión educativa del último medio siglo. [Tesis Doctoral]. Madrid: Universidad Complutense.

McIntyre, A. (2009). The very Idea of a University: Aristotle, Newman and us. Brithish Journal of Educational Studies, 57 (4), 347-362. doi: http://dx.doi. org/10.1111/j.1467-8527.2009.00443.x

McIntyre, A. (2012). Dios, filosofía, universidades. Granada: Nuevo Inicio.

McIntyre, K. (2013). Liberal Education and the Teleological Question; or Why Should a Dentist Read Chaucer? Journal of Philosophy of Education, 47(3), 341-363. doi: http://dx.doi. org/10.1111/1467-9752.12003

Miller, E.J. (2011). John Henry Newman's Idea of Alma Mater. Newman Studies, 8(2), 19-28. doi: 10.5840/nsj20118221

Minogue, K. (2005). The Concept of a University. London: Transaction Publishers. 
Mlinar, A. (2013). John Henry Newman on University: Actuality of 160 Year Old Discourse. Shynthesis Philosophica, 55-56(1-2), 131-148.

Newman, J.H (1900). A Letter Addressed to the Duke of Norfolk on Occasion of Mr. Gladstone's Recent Expostulation. London: Longmans, Green. Recuperado de https://goo.gl/HgsGAK

Newman J.H. (1903). An Essay in Aid of a Grammar of Assent. London: Longmans, Green. Recuperado de https://goo.gl/27nyBv

Newman J.H. (1908). Apología pro Vita Sua (1865). London: Longmans, Green. Recuperado de https://goo.gl/ V4pkir

Newman J.H. (1907b). Discussions and Arguments on Various Subjects (The Tamworth reading room). London: Longmans, Green. Recuperado de https://goo.gl/qnL1Ha

Newman J.H. (1909b). Historical Sketches, vol. III, (The Rise and Progress of Universities). London: Longmans, Green. Recuperado de https://goo.gl/YHd29R

Newman J.H. (1907). Idea of a University. London: Longmans, Green. Recuperado de https://goo.gl/Jjg2BJ

Newman J.H. (1909). Oxford University Sermons (Implicit and Explicit Reason). London: Longmans, Green. Recuperado de https://goo.gl/YKuf9t

Newman J.H. (1908b). Sermons Preached in Various Occasions (Intellect, the instrument of Religious Training). London: Longmans, Green. London: Longmans, Green. Recuperado de https://goo.gl/9UctJ3
Nubiola, J. (2008). La transformación de la universidad española. Nueva Revista de política, cultura y arte, 120, 81-89.

Ortega y Gasset, J. (2007). Misión de la universidad. Madrid: Alianza Editorial.

Polo, L. (1998). Antropología trascendental. La persona humana. Tomo I. Pamplona: Eunsa.

Ramón y Cajal, S. (2015). Los tónicos de la voluntad. Reglas y consejos sobre la investigación científica. Madrid: Gadir.

Reyero, D. (2014). La excelencia docente universitaria. Análisis y propuestas para una mejor evaluación del profesorado universitario. Educación XXI, 2, 125-143, doi: 10.5944/educxx1.17.2.11482

Rupert, M.J. (1998). John Henry Newman on Education. Ann Arbor: Proquest Company.

Shulman, L. S. (1986). Those who Understand: Knowledge Growth in Teaching. Educational Researcher, 15(2), doi: 10.3102/0013189X015002004

Tillman, M.K. (2008). A Rethoric in Conduct: The Gentleman of the University and the Gentleman of the Oratory. Newman Studies, 5(2), pp. 6-25.

Torralba, J.M (2013). La idea de educación liberal. De cómo se inventaron las humanidades. En J. Arana Cañedo-Argüelles (Coord.), Falso saberes. La suplantación del conocimiento en la cultura contemporánea, (pp. 65-78). Madrid: Biblioteca Nueva.

Waldmann, T. (1967) Personal Influence According to John Henry Newman. Ann Arbor: Proquest Company. 


\section{PERFIL ACADÉMICO Y PROFESIONAL DEL AUTOR}

Miguel Rumayor. Profesor Titular de Filosofía de la Educación en la Escuela de Educación de la Universidad Panamericana (Guadalajara, México). También es profesor del Centro Universitario Villanueva (Madrid, España) y director del Departamento de Pedagogía. Forma parte del Sistema Nacional de investigadores de México. Acreditado en ANECA como Profesor Titular. Tiene reconocidos dos sexenios de investigación por la CENAI. Ha sido Visiting Fellow de las universidades de Oxford, Notre Dame, Catholic University y Duquesne University.

Dirección del Autor: Centro Universitario Villanueva Costa Brava 2

28034 Madrid

E-mail: mrumayor@up.edu.mx

Fecha recepción del artículo: 22. Octubre. 2017

Fecha aceptación del artículo: 03. Marzo. 2018

Fecha revisión para publicación: 20. Junio. 2018 